\title{
$\beta$-Hydroxybutyrate Mitigated Heart Failure with Preserved Ejection Fraction by Increasing Treg Cells via Nox2/GSK-3 $\beta$
}

\author{
Shengen Liaol,* \\ Yuan Tang ${ }^{1} *$ \\ Xin Yue ${ }^{\prime}$ \\ Rongrong Gao' \\ Wenming Yao' \\ Yanli Zhou' \\ Haifeng Zhang ${ }^{2,3}$ \\ 'Department of Cardiology, The First \\ Affiliated Hospital of Nanjing Medical \\ University, Jiangsu Province Hospital, \\ Nanjing, 2 I0029, People's Republic of \\ China; ${ }^{2}$ Department of Cardiology, The \\ Affiliated Suzhou Hospital of Nanjing \\ Medical University, Suzhou Municipal \\ Hospital, Gusu School, Nanjing Medical \\ University, Suzhou, 215002, People's \\ Republic of China; ${ }^{3}$ Department of \\ Cardiology, Jiangsu Province Hospital, \\ Nanjing, 210029, People's Republic of \\ China
}

*These authors contributed equally to this work
Correspondence: Haifeng Zhang

Department of Cardiology, The Affiliated Suzhou Hospital of Nanjing Medical University, Suzhou Municipal Hospital, Gusu School, Nanjing Medical University, Suzhou, 215002, People's Republic of China

Email haifeng_zhang@163.com
Background: This study was designed to investigate the cardioprotective role of $\beta$ hydroxybutyrate (BHB) in heart failure with preserved ejection fraction (HFpEF) and the underlying mechanism.

Methods: A two-hit model with a high-fat diet (HFD) and $\mathrm{N}^{\omega}$-nitrol-arginine methyl ester (L-NAME) was used as an HFpEF model. The treatment group received a weekly intraperitoneal injection of $\beta$-hydroxybutyrate (BHB). Cardiac function, inflammation, and fibrosis were evaluated. $\mathrm{CD}^{+} \mathrm{CD}^{+}{ }^{+}$Foxp $3{ }^{+}$positive cells within the myocardium were quantified by flow cytometry. The NADPH oxidase 2 (NOX2)/glycogen synthase kinase-3 $\beta$ (GSK3 $\beta$ ) pathway was examined by immunoblot analysis.

Results: BHB improved diastolic function, fibrosis and cardiac remodeling in HFpEF. Additionally, BHB inhibited cardiac inflammation and increased cardiac Treg cells, which could be due to the downregulation of the NOX2/GSK-3 $\beta$ pathway.

Conclusion: BHB protected against the progression of HFpEF by increasing cardiac Treg cells by modulating the NOX2/GSK-3 $\beta$ pathway.

Keywords: $\beta$-hydroxybutyrate, HFpEF, Treg cells, NOX2, GSK-3 $\beta$

\section{Introduction}

Heart failure (HF) is a leading cause of morbidity and mortality worldwide. The prevalence of heart failure with preserved ejection fraction (HFpEF) is approximately $2 \%$, but its prevalence is over $50 \%$ in HF. HFpEF may become the predominant form of $\mathrm{HF}$ in the future owing to the aging population. ${ }^{1}$ The early Framingham Heart Study reported that patients with HFpEF had an annual mortality rate of $8.7 \% .^{2}$ The primary mechanism was systemic abnormalities, such as metabolic stress, hypertension and aging. ${ }^{3}$ In addition, some cardiac and noncardiac factors promoted endothelial dysfunction and exercise intolerance in HEpEF. However, effective therapies that are specific for HFpEF are still lacking. ${ }^{5}$

$\beta$-Hydroxybutyrate $(\mathrm{BHB})$ is a type of ketone body that is produced predominantly in the liver of mammals. In addition to metabolic effects, it is known to play an immunomodulatory role by ligating hydroxycarboxylic acid receptor $2^{6}$ and inhibiting inflammasome activation and histone deacetylase expression. ${ }^{7}$ Studies have suggested that increased myocardial utilization of BHB was observed in advanced HF patients, ${ }^{8}$ and increased ketone body oxidation may mitigate HF disease progression. ${ }^{9}$ Deletion of succinyl-CoA:3-oxoacid coenzyme A transferase, a ketolytic rate-limiting enzyme, 
resulted in the elevation of circulating BHB and protected against the development of HF. ${ }^{10}$ It has been hypothesized that the cardioprotective role of sodium-glucose cotransporter 2 inhibitors may be attributed to increased circulating BHB levels. ${ }^{11}$ BHB has beneficial hemodynamic effects in HF patients with reduced EF without impairing myocardial external energy efficiency. ${ }^{12}$

It was reported that BHB ameliorated mitochondrial hyperacetylation and NLR family pyrin domain containing 3 (NLRP3) inflammasome information based on a HFpEF model. ${ }^{13}$ Using a newly established "two-hit" mouse model of $\mathrm{HFpEF}^{14}$ that uses a combination treatment of HFD+L-NAME for fifteen weeks, we investigated the role of $\mathrm{BHB}$ in the progression of $\mathrm{HF}$ and the underlying mechanisms.

\section{Methods and Materials}

\section{Animals}

Male C57BL/6N mice (6 to 8 weeks old) were purchased from the Institute of Model Animals of Nanjing University (Nanjing, China). Mice were maintained on a 12-h light/ dark cycle and had free access to food. They were randomly divided into three groups: the sham group, HF group, and BHB group. In the HF group, mice were fed a high-fat diet (D12494, Research Diet) and were given drinking water containing $\mathrm{N}^{\omega}$-nitrol-arginine methyl ester (L-NAME, $0.5 \mathrm{~g} / \mathrm{L}$, Sigma) for 15 weeks. ${ }^{14}$ In the BHB group, $\beta$-hydroxybutyrate (Sigma, USA) was additionally administered to mice at a dose of $10 \mathrm{mmol} / \mathrm{kg}$ once per week via intraperitoneal injections. Each group had five mice, and five mice were used for the following biochemical and histological analyses. All procedures were approved by the Institutional Animal Care and Use Committee (IACUC 1803019) of Nanjing Medical University and were in accordance with the Guide for the Care and Use of Laboratory Animals (National Academies Press, 2011).

\section{Echocardiography}

After 15 weeks, the cardiac function of all groups was evaluated using transthoracic echocardiography (VisualSonics Vevo 2100, Japan). Anesthesia was induced with $5 \%$ isoflurane until there was no response to tail touch. Indices of systolic function were obtained from short-axis M-mode scans, while indices of diastolic function were obtained from apical four-chamber views. The parameters collected included ejection fraction (LVEF), fractional shortening (FS), peak Doppler blood inflow velocity across the mitral valve during early diastole (E), peak Doppler blood inflow velocity across the mitral valve during late diastole (A), left ventricular internal endsystolic diameter (LVIDs), and left ventricular internal end-diastolic diameter (LVIDd). All parameters were measured at least three times and averaged.

\section{Tail-Cuff Blood Pressure Recordings}

Systolic blood pressure was measured noninvasively using the tail-cuff method (CODA, Kent Scientific). Mice were placed in individual holders, and measurements were performed under steady-state conditions. Before testing, all mice were trained to become accustomed to short-term restraint. Blood pressure was recorded at least 10 times, and the readings were averaged.

\section{Gravimetric Analysis}

Mice were sacrificed by cervical dislocation after anesthesia with tiletamine $(0.09 \mathrm{mg} / \mathrm{g})$, zolazepam $(0.09 \mathrm{mg} / \mathrm{g})$, and $0.01 \%$ xylazine $(0.04 \mathrm{~mL} / \mathrm{g})$. The hearts and lungs were removed from the chest cavity and washed in phosphate buffered saline. The hearts and lungs were blotted with paper and weighed. The lower limbs were dissected, and the length of the left tibia was measured. The lung was incubated in a $70{ }^{\circ} \mathrm{C}$ oven to dry for 24 hours and reweighed to calculate the wet-to-dry ratio. Heart weight and lung weight were indicative of cardiac hypertrophy and pulmonary congestion, respectively, and served as preclinical surrogates of HF.

\section{Histology}

Hearts were collected and fixed in 4\% paraformaldehyde overnight and processed for routine paraffin histology. The $5-\mu \mathrm{m}$ sections were stained with hematoxylin-eosin and picrosirius red. Wheat germ agglutinin (WGA) staining was used to evaluate the cross-sectional area of cardiomyocytes. To visualize ROS production within the myocardium, fresh hearts were immediately embedded in O.C. T. compound (Servicebio, Wuhan) and cut into $5 \mu \mathrm{m}$ slices. Slices were stained with $5 \mu \mathrm{M}$ dihydroethidium (DHE, Beyotime, Nantong) for 1 hour in the dark at room temperature and then washed 3 times for 1 hour with Krebs-HEPES buffer. For apoptosis observation, heart slices were stained using a TUNEL apoptosis assay kit (Beyotime, Nantong). For immunofluorescence staining, heart slices were incubated overnight with a CD68 antibody (anti-CD68, Abcam, USA) and subsequently 
fluorescence-labeled with a secondary antibody. Images were viewed with confocal scanning microscopy (Zeiss, LSM 800), and the fluorescence intensity was estimated using ImageJ (NIH, USA).

\section{Flow Cytometry}

The excised hearts were dissociated in PBS with a syringe plunger to obtain a single-cell suspension. Cells were subsequently stained with CD3-APC and CD4-FITC at 4 ${ }^{\circ} \mathrm{C}$ for 15 minutes and then permeabilized (Transcription Factor Buffer Set, Thermo Fisher, USA) to stain for Foxp3-PE (eBiosciences, USA). After washing three times, the cells were analyzed by flow cytometry (BD FACSAria, USA). The FACS data were then analyzed with FlowJo software (Treestar, USA).

\section{Quantitative Real-Time Polymerase Chain Reaction (qPCR)}

Total RNA was extracted from mouse hearts using RNAiso plus (TaKaRa; Japan) according to the manufacturer's instructions. A total of 1000 ng RNA was used for reverse transcription using HiScriptII Q RT SuperMix (Vazyme; China). qPCR reactions were performed in triplicate with ChamQ SYBR qPCR Master Mix (Vazyme; China) according to the manufacturer's protocol. The results were normalized to GAPDH using the $2^{-\Delta \Delta \mathrm{Ct}}$ relative quantification method. The following PCR primer sequences were used for each gene (forward, reverse sequences): GAPDH AGGTCGGTGTGAACGGATTTG, TGTAGACCATGTAGTTGAGGTCA; IL-1b GCAACTG TTCCTGAACTCAACT， ATCTTTTGGGGTCCGTCAA CT; IL-6 TAGTCCTTCCTACCCCAATTTCC, TTGGTC CTTAGCCACTCCTTC; IL-10 GCTCTTACTGACTGGC ATGAG, CGCAGCTCTAGGAGCATGTG; a-SMA GTC CCAGACATCAGGGAGTAA, TCGGATACTTCAGCGT CAGGA; ANP TCGTCTTGGCCTTTTGGCT, TCCAGG TGGTCTAGCAGGTTCT; BNP AAGTCCTAGCCAGTC TCCAGA, GAGCTGTCTCTGGGCCATTTC.

\section{Immunoblotting}

Total protein was extracted from frozen heart tissues using RIPA buffer containing proteinase and phosphatase inhibitors (Roche; Germany). Protein lysates were separated by SDS-PAGE and transferred to PVDF membranes (Millipore; USA). The membranes were incubated with the following primary antibodies: GSK-3b, pS9-GSK-3b, NOX2 and GAPDH (Abcam; USA) overnight at $4{ }^{\circ} \mathrm{C}$ and then incubated with goat anti-rabbit IgG (Abcam; USA). The Western blot bands were detected using an ECL kit (Keygene; China). ImageJ (NIH; USA) was used to quantify the specific protein expression levels.

\section{Statistical Analysis}

Data are mean \pm standard deviation. Differences were analyzed by two-tailed unpaired Student's $t$-test for experiments with two groups and one-way ANOVA for multiple comparisons. A value of $P<0.05$ was considered statistically significant. All experiments were performed with three biological replicates. Statistical analyses were conducted using GraphPad Prism software 8.0.

\section{Results}

BHB Alleviated the Diastolic Dysfunction in HFpEF Mice

At the 15th week of the experimental protocol, transthoracic echocardiography was performed to evaluate cardiac function. Representative echocardiography images indicating diastolic function are shown in Figure 1A. As expected, treatment with HFD and L-NAME had no effect on the ejection fraction (EF, Figure 1B) and fractional shortening (FS, Figure 1C) but decreased the E/A ratio (Figure 1D). However, BHB treatment rescued this decrease in the E/A ratio. In addition, systolic blood pressure was lower in the BHB group than in the HF group $(\mathrm{P}<0.01$, Figure 1E).

\section{BHB Inhibited Cardiac Remodeling and Fibrosis}

The HFpEF model was successfully established based on a previous method. Therefore, our study focused on the therapeutic effect of BHB on HFpEF mice. As shown in Figure 2A, BHB treatment reduced heart weight/tibia length $(\mathrm{P}<0.01)$ and lung weight wet/dry ratio, even though the latter was not significantly changed. These results suggest that BHB alleviated cardiac hypertrophy and pulmonary congestion. qPCR results also demonstrated that heart failure-associated mRNAs, including ANP, BNP $(\mathrm{P}<0.05)$ and a-SMA $(\mathrm{P}<0.05)$, were downregulated by $\mathrm{BHB}$ treatment (Figure $2 \mathrm{~B}$ ). In addition, BHB decreased the expression of inflammatory genes, including IL-1b $(\mathrm{P}<0.05)$, IL-6 $(\mathrm{P}<0.01)$ and IL-10 $(\mathrm{P}<0.01)$ (Figure $2 \mathrm{C}$ ). HFpEF was characterized by increased cardiac fibrosis and cardiomyocyte sizes. Our results revealed that BHB restored broken myofilaments 


\section{A Sham}

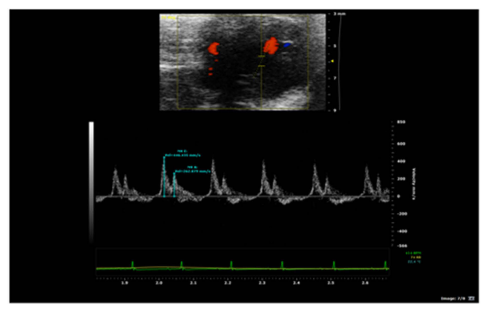

HF

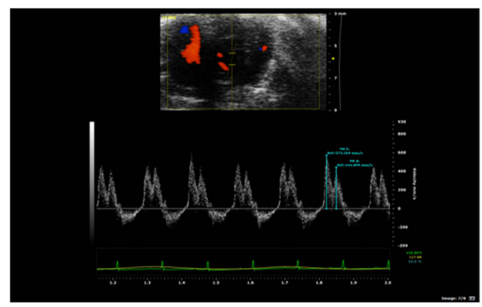

BHB

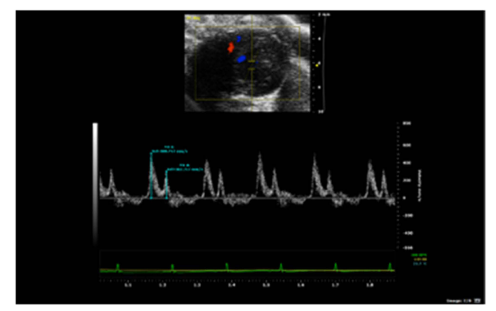

B

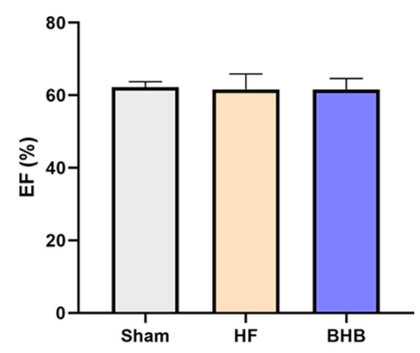

C

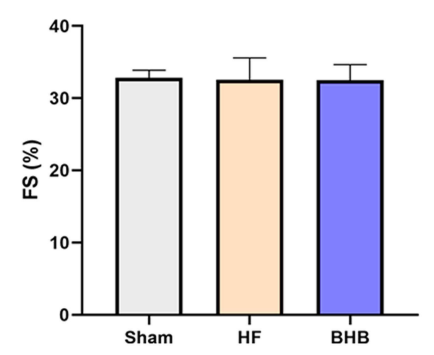

D

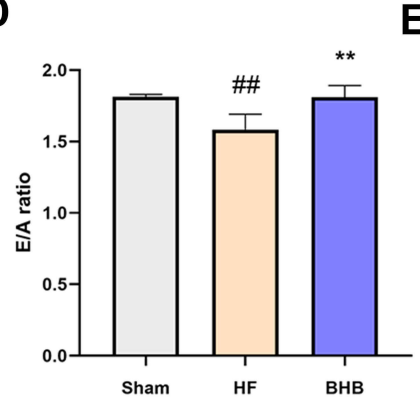

E

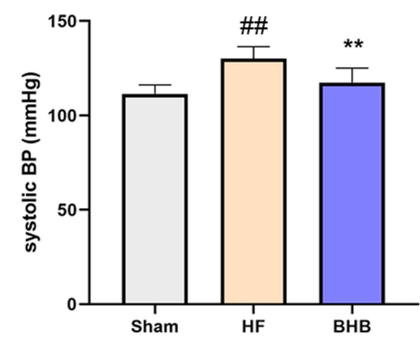

Figure I BHB improved cardiac diastolic dysfunction in HFpEF mice. C57B/6 male mice were divided into three groups: Sham, HF, and BHB group. (A) Representative echocardiographic images between groups. (B) Left ventricular ejection fraction (EF, \%). (C) Left ventricular fraction shortening (FS, \%). (D) E/A ratio. (E) systolic blood pressure (systolic BP, mmHg). $n=5$ mice/group. ${ }^{\#} \mathrm{p}<0.01$ vs sham; ${ }^{* *} \mathrm{p}<0.01$ vs HF.

and disordered sarcomeres (Figure 3A). In addition, $\mathrm{BHB}$ reduced the percentage of interstitial fibrosis (Figure 3B) and cardiomyocyte sizes (Figure 3C).

\section{BHB Prevented Cardiomyocyte Apoptosis, Cardiac Inflammation and Oxidative Stress}

Increases in oxidative stress and the inflammatory response contributed to cardiomyocyte apoptosis in HFpEF. As shown in Figure 4A, the fluorescence intensity of TUNEL staining was decreased by BHB treatment. In addition, there was a significant reduction in the intensity of DHE staining in the BHB group (Figure 4B). F4/80 immunofluorescence showed that $\mathrm{BHB}$ decreased the percentage of macrophage infiltration (Figure 4C). These results demonstrate that BHB could inhibit cardiomyocyte apoptosis by mitigating excess oxidative stress and cardiac inflammation.

\section{BHB Increased the Number of Treg Cells Within the Myocardium}

Treg cells are anti-inflammatory cells that play a cardioprotective role in HFpEF hearts. As shown in
Figure 5, we found that $\mathrm{BHB}$ increased the number of $\mathrm{CD} 3{ }^{+} \mathrm{CD} 4^{+} \mathrm{Foxp}^{+}$cells, suggesting an increased number of Treg cells within the myocardium.

\section{BHB Played a Cardioprotective Role by Downregulating the NOX2/GSK3 $\beta$ Pathway}

Previous studies found that NOX2 promoted the phosphorylation of serine 9 of GSK $3 \beta,{ }^{15}$ which is associated with heart failure. ${ }^{16}$ Therefore, we measured the expression of NOX2/GSK $3 \beta$ in failing hearts. As shown in Figure 6, immunoblot results showed that BHB inhibited the expression of NOX2 and pS9-GSK3 $\beta(\mathrm{P}<0.001)$.

\section{Discussion}

We found that HFD and L-NAME treatment increased systolic blood pressure, decreased cardiac diastolic function, and increased systemic and cardiac inflammation, which recapitulated the numerous systemic and cardiovascular features of human HFpEF. Our study showed that BHB treatment could mitigate diastolic dysfunction and decrease systolic blood pressure in HFpEF. In addition, 
A

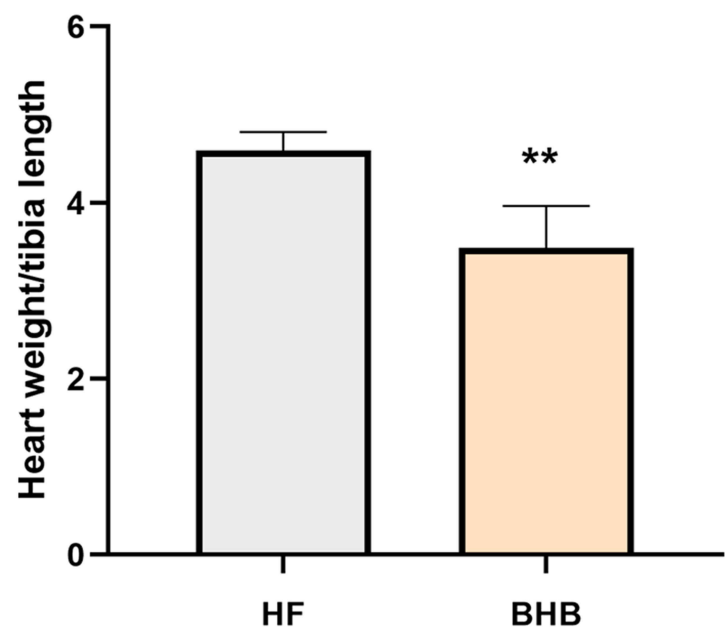

B

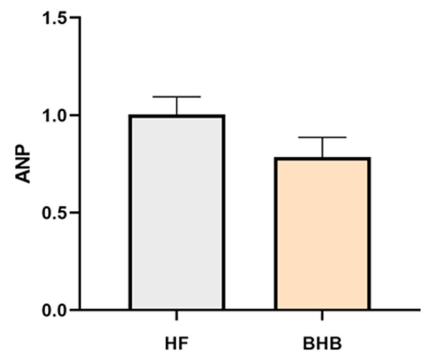

C

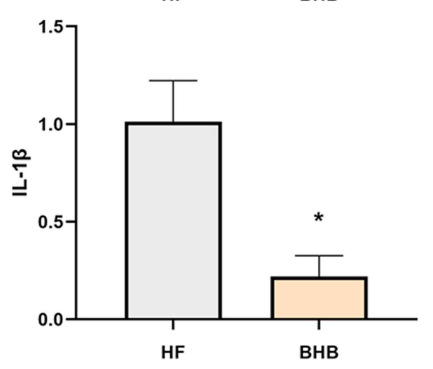

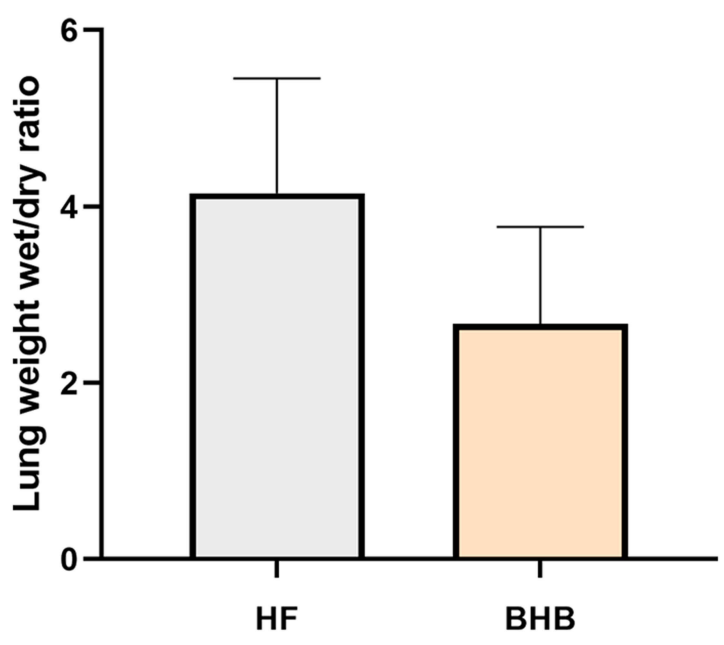
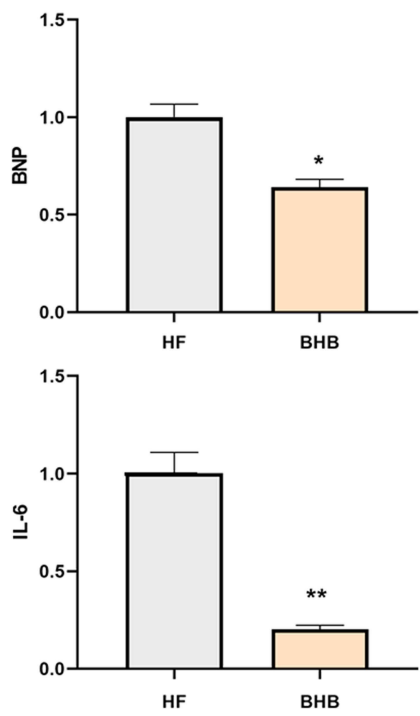
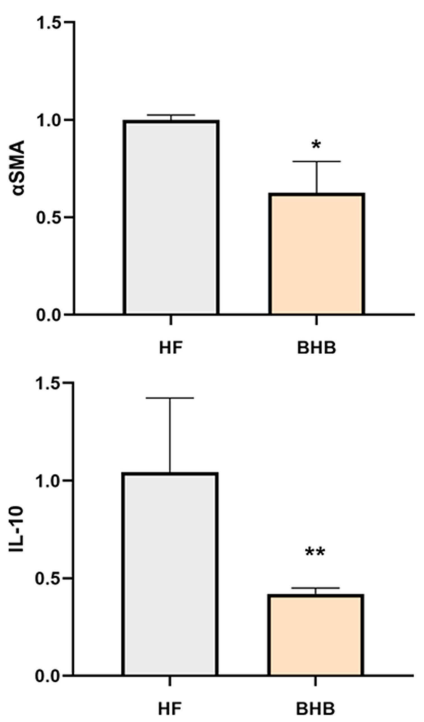

Figure 2 BHB improved cardiac inflammation in HFpEF mice. (A) The heart weight/tibia length ratio and lung weight wet/dry ratio were measured. (B) The fibrotic mRNA expression of ANP, BNP and $\alpha$ SMA were measured using qPCR. (C) The inflammatory mRNA expression of IL-I $\beta$, IL- 6 and IL-I0 were measured using qPCR. * $<<0.05$, $* * p<0.01$.

BHB alleviated cardiac inflammation and cardiac remodeling, which could be related to an increased number of Treg cells by downregulating the NOX2/GSK3 $\beta$ pathway.

Recent investigations have suggested that failing hearts shift to increased ketone consumption as a fuel. ${ }^{17,18}$ Ketone treatment improved cardiac function in preclinical models of heart failure ${ }^{19,20}$ and human heart failure. ${ }^{8}$ BHB, a ketone body, has been traditionally regarded as an alternative carrier of energy and has proven beneficial effects on cardiovascular diseases. ${ }^{21}$ Specifically, BHB could protect against oxidative stress $^{22}$ and inhibit NLRP3 inflammasome ${ }^{13}$ in failing hearts. In line with previous studies on the anti-inflammatory and antioxidative roles of BHB, our results show that BHB treatment counteracted proinflammatory cytokine-induced dysfunction and fibrosis, thus protecting against the pathogenesis of HFpEF.

Sustained inflammation was a hallmark of chronic heart failure. Studies found that mice with ischemic HF exhibited a higher presence of Th1 and Th17 cells than Th2 and Treg cells. ${ }^{23}$ The Th17/Treg imbalance also existed in patients with HFpEF. ${ }^{24}$ Decreased Tregs were reported to aggravate myocardial fibrosis and $\mathrm{HF}^{25}$ and upregulation of Tregs could ameliorate cardiac dysfunction in chronic heart failure. ${ }^{25}$ Our results also show that BHB treatment increased Treg cells and inhibited cardiac inflammation. These results suggest that BHB not only serves as an energy fuel but also functions as an immunomodulatory agent. 
A
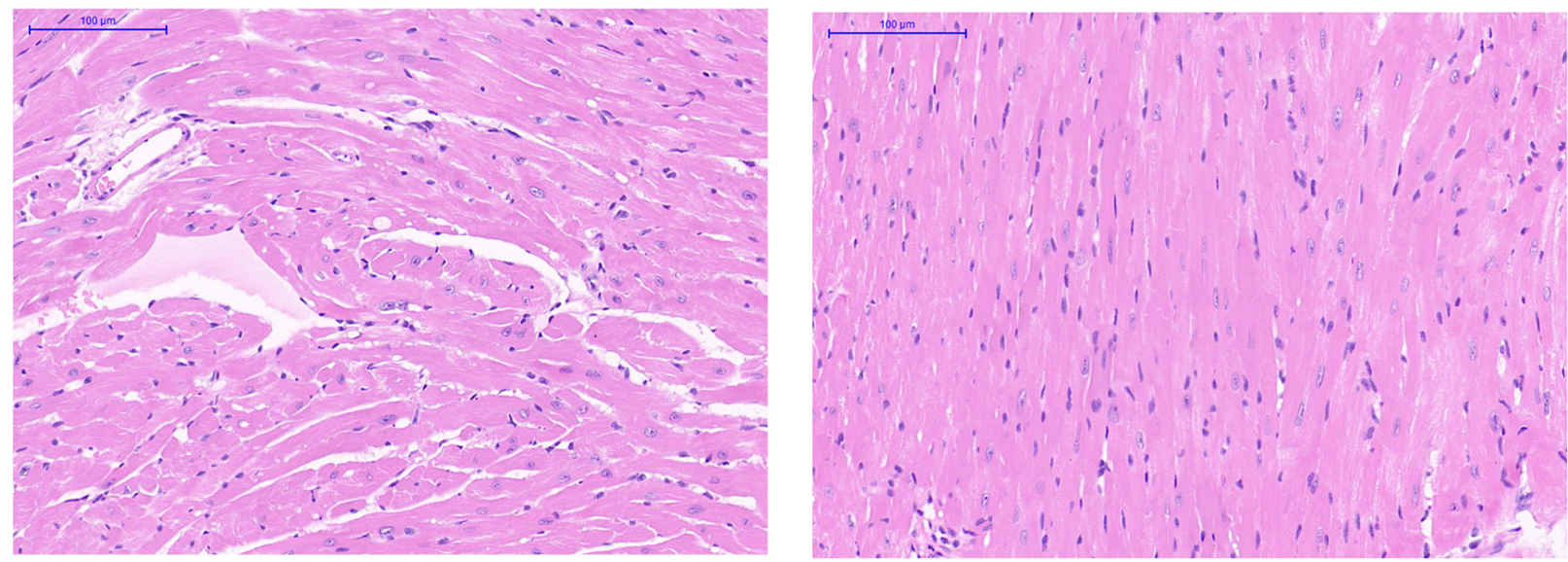

B
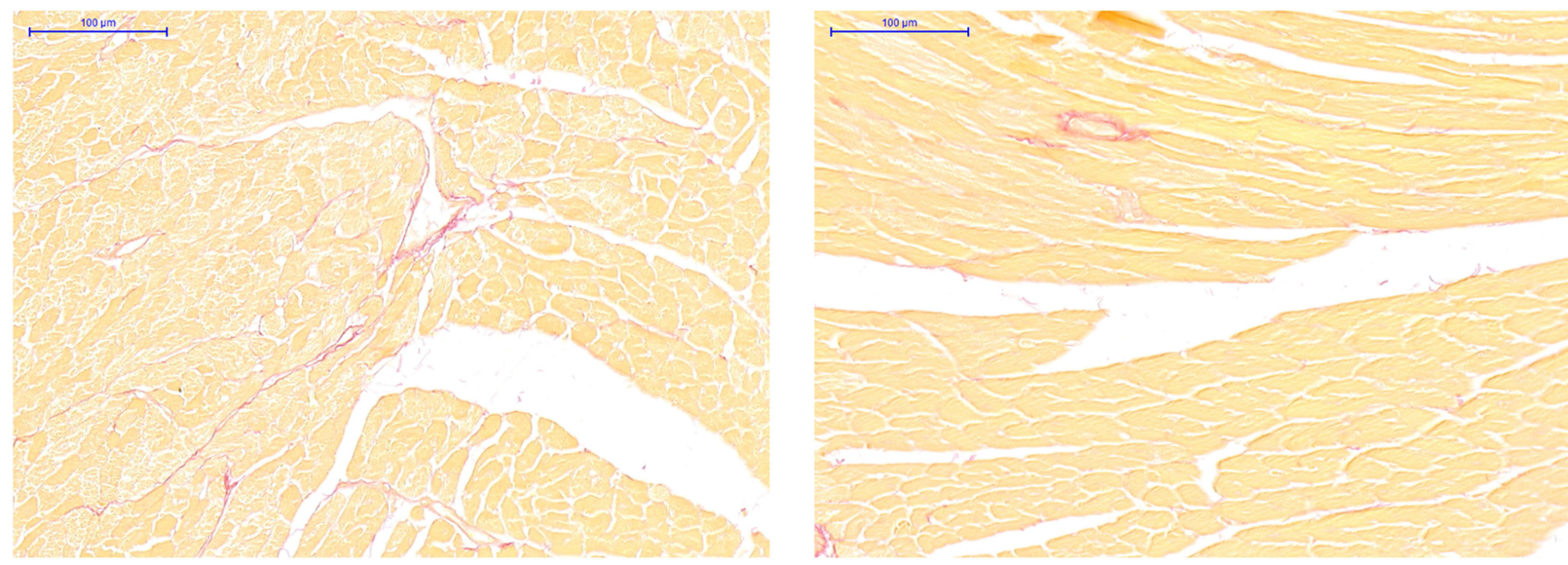

C
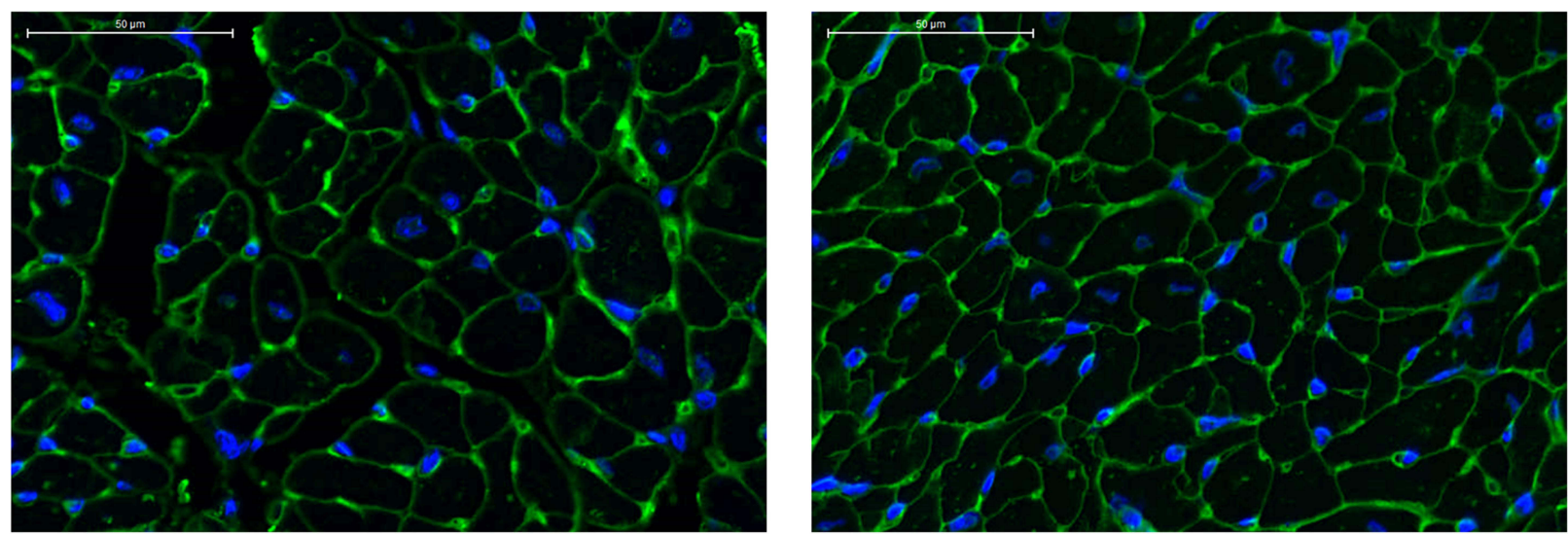

Figure 3 BHB protected from cardiac remodeling in vivo. (A) The HE staining was performed for the structure alternations. (B) Cardiac fibrosis was evaluated using Masson staining. (C) Cell size was showed using WGA staining.

Studies have found that NOX2 inhibits Treg cells and aggravates cardiac remodeling, and the deletion of NOX2 increases cardiac Treg cells. ${ }^{26}$ In addition, knockout of NOX2 inhibited the phosphorylation of serine-9 of GSK$3 \beta .{ }^{15}$ GSK-3 $\beta$ is a serine-threonine kinase and participates in many cell functions, eg, transcription, metabolism, growth and apoptosis. ${ }^{27}$ GSK-3 $\beta$ was reported to be involved in heart failure. ${ }^{28}$ Studies found that S9 phosphorylation of GSK-3 $\beta$ mediated pathological hypertrophy in $\mathrm{HF}^{29}$ In line with the above results, we demonstrated that NOX2 and S9-GSK-3 $\beta$ 
A

HF

BHB

B

HF

BHB

DAPI

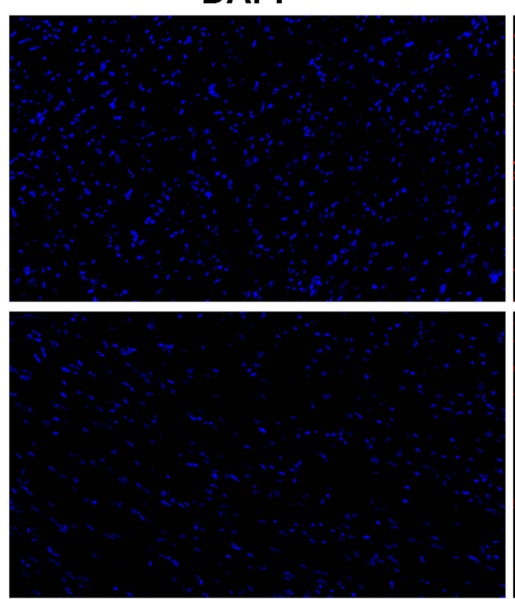

C

HF
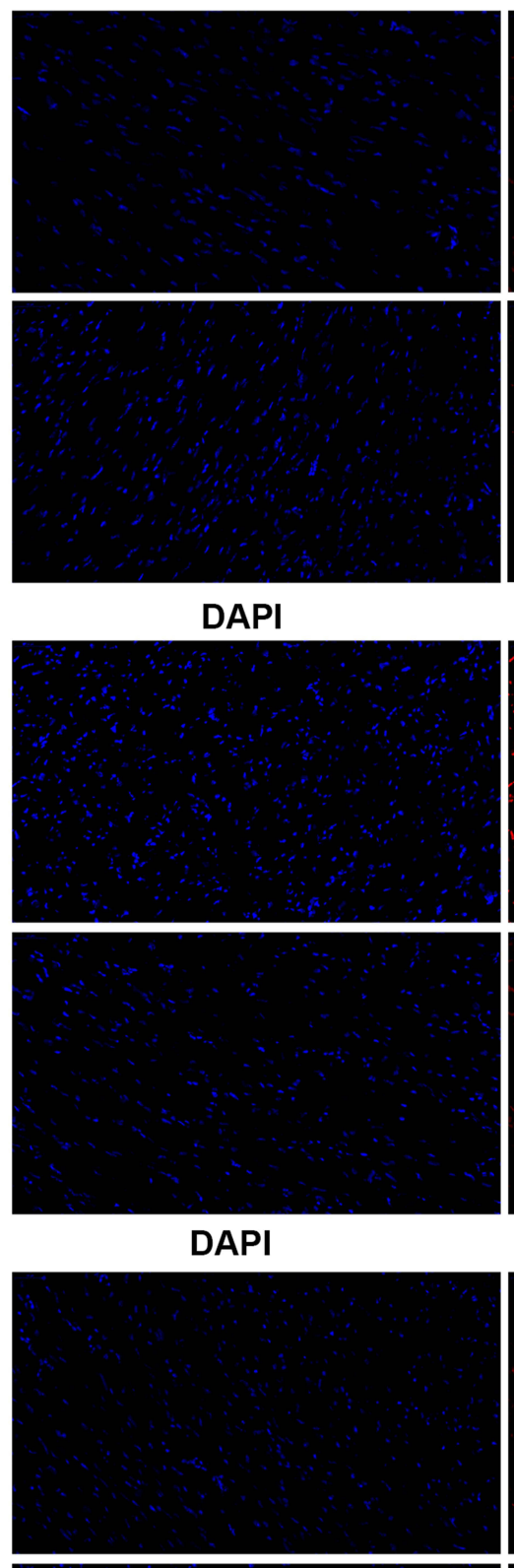

TUNEL

Merge
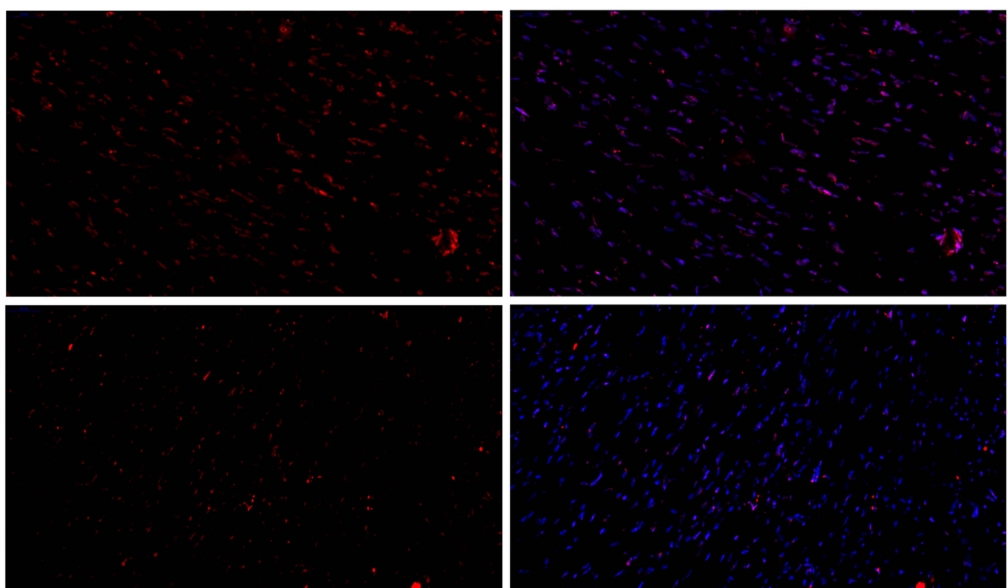

4-HNE

Merge

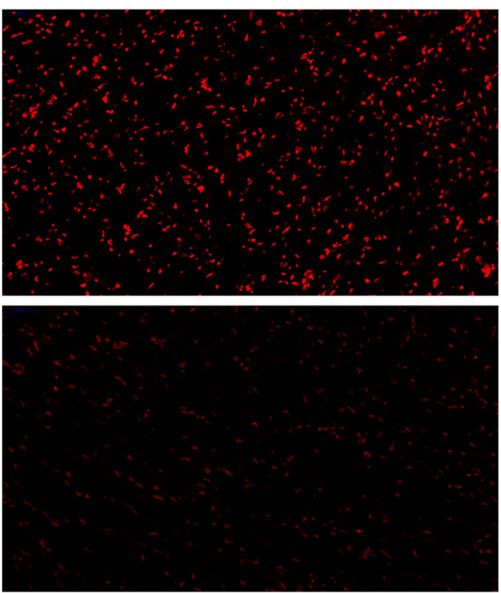

$F 4 / 80$

Merge
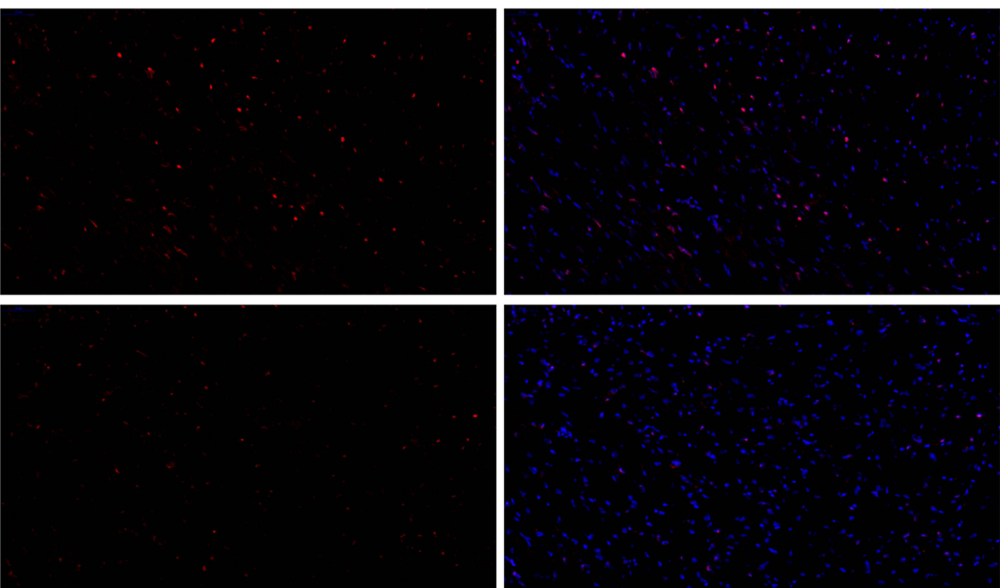

Figure 4 BHB prevented from cardiomyocyte apoptosis and macrophage infiltration. (A) DHE staining, (B) TUNEL staining and (C) F4/80 immunofluorescence were performed between groups.

were increased in the HFpEF group, which could be reversed by BHB treatment. BHB could increase Tregs and provide a cardioprotective role by regulating the NOX $2 /$ GSK-3 $\beta$ pathway. Therefore, a ketogenic diet or short-chain fatty acid supplements could be potential treatments for patients with HFpEF.

Some limitations of this study indicate that these results should be interpreted with caution. First, invasive 

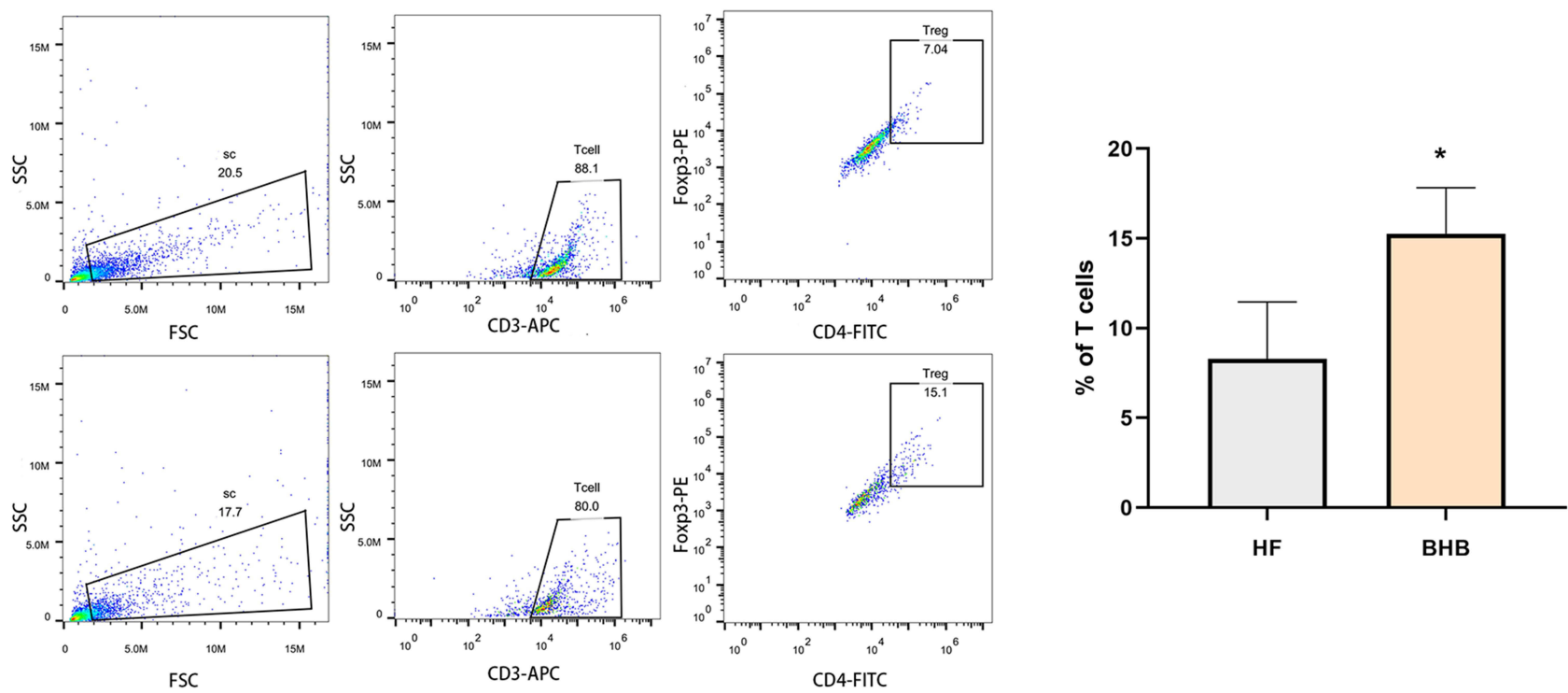

Figure 5 BHB increased the percentage of Treg cells within myocardium. Cardiac Treg cells $\left(\mathrm{CD} 3^{+} \mathrm{CD} 4^{+}\right.$Foxp $\left.3^{+}\right)$were examined using flow cytometry. ${ }^{*}<0.05$.
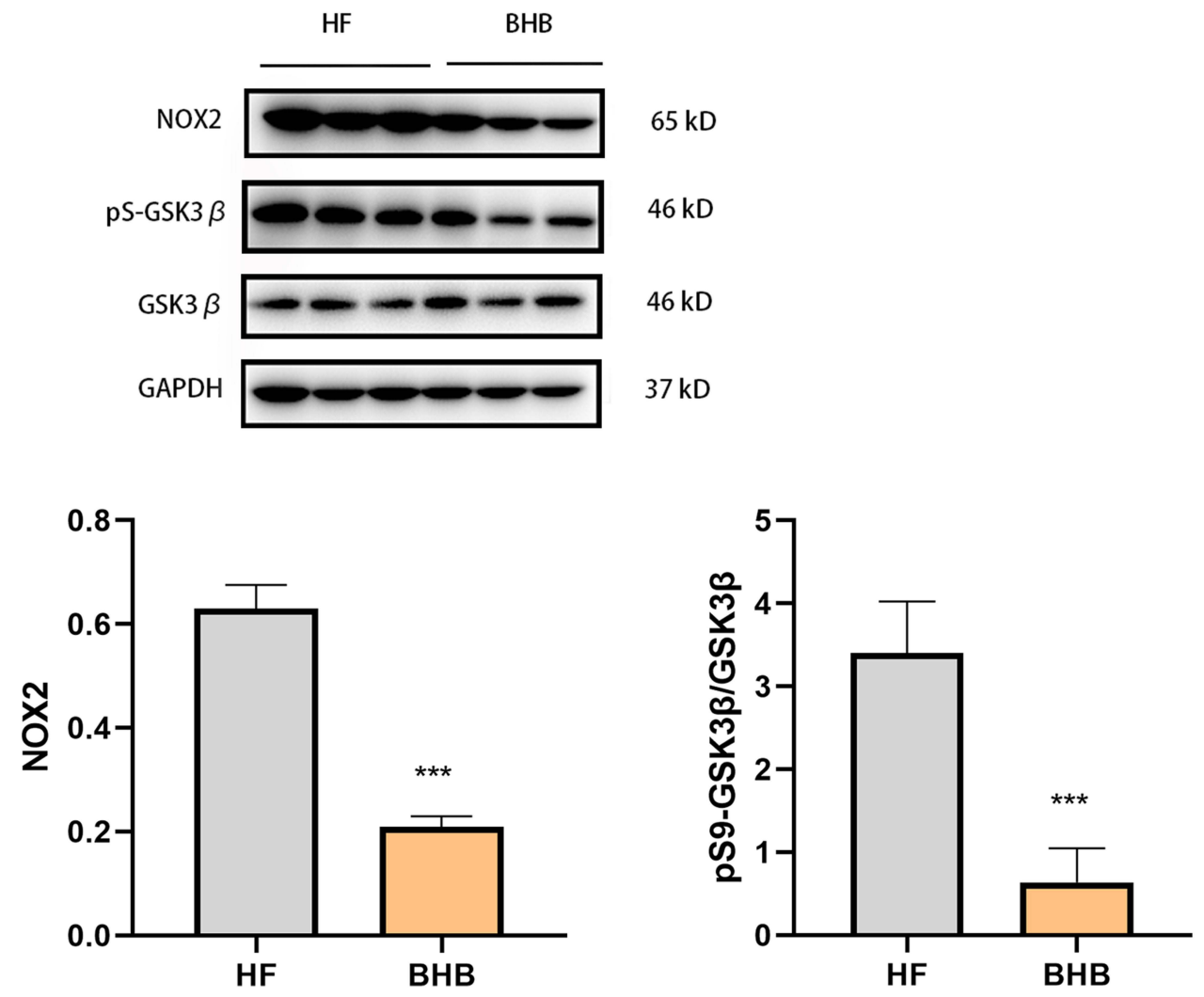

Figure 6 BHB inhibited the GSK3 $\beta / N O X 2$ pathway. The protein expression of NOX2, GSK3 $\beta$ and pS9-GSK3 $\beta$ were measured by Western blot. Below were the quantification results among groups. $* * * p<0.001$.

examinations for cardiac function, such as pressure-volume analysis, were not performed. Second, more experiments, such as overexpression or silencing of the NOX2/GSK-3 $\beta$ pathway, should be performed to validate the molecular mechanism.

\section{Conclusions}

Our results found that BHB could increase cardiac Treg cells and inhibit cardiac inflammation, thereby protecting against the progression of HFpEF. 


\section{Data Sharing Statement}

All data are available from the corresponding author upon reasonable request.

\section{Ethical Approval}

All procedures were approved by the Institutional Animal Care and Use Committee (IACUC 1803019) of Nanjing Medical University and were in accordance with the Guide for the Care and Use of Laboratory Animals (National Academies Press, 2011).

\section{Author Contributions}

All authors made the following substantial contributions: conception and design, acquisition of data, or analysis and interpretation of data; took part in drafting the article or revising it critically for important intellectual content; agreed to submit to the current journal; gave final approval of the version to be published; and agree to be accountable for all aspects of the work.

\section{Funding}

This work was supported by a project from Gusu School (Grant No. GSRCKY20210204).

\section{Disclosure}

Shengen Liao and Yuan Tang are co-first authors for this study. The authors declare no potential conflicts of interest with respect to the research, authorship, and/or publication of this article.

\section{References}

1. Cho DH, Yoo BS. Current prevalence, incidence, and outcomes of heart failure with preserved ejection fraction. Heart Fail Clin. 2021;17 (3):315-326. doi:10.1016/j.hfc.2021.03.002

2. Steinberg BA, Zhao X, Heidenreich PA, et al. Trends in patients hospitalized with heart failure and preserved left ventricular ejection fraction: prevalence, therapies, and outcomes. Circulation. 2012;126 (1):65-75. doi:10.1161/CIRCULATIONAHA.111.080770

3. Shah SJ, Kitzman DW, Borlaug BA, et al. Phenotype-specific treatment of heart failure with preserved ejection fraction: a multiorgan roadmap. Circulation. 2016;134(1):73-90. doi:10.1161/CIRCULATIONAHA. 116.021884

4. Gevaert AB, Boen JRA, Segers VF, et al. Heart failure with preserved ejection fraction: a review of cardiac and noncardiac pathophysiology. Front Physiol. 2019;10:638. doi:10.3389/fphys.2019.00638

5. Gentile F, Ghionzoli N, Borrelli C, et al. Epidemiological and clinical boundaries of heart failure with preserved ejection fraction. Eur J Prev Cardiol. 2021;zwab077. doi:10.1093/eurjpc/zwab077

6. Youm YH, Nguyen KY, Grant RW, et al. The ketone metabolite beta-hydroxybutyrate blocks NLRP3 inflammasome-mediated inflammatory disease. Nat Med. 2015;21(3):263-269. doi:10.1038/ nm.3804
7. Chang PV, Hao L, Offermanns S, et al. The microbial metabolite butyrate regulates intestinal macrophage function via histone deacetylase inhibition. Proc Natl Acad Sci U S A. 2014;111(6):2247-2252. doi:10.1073/pnas.1322269111

8. Bedi KC, Snyder NW, Brandimarto J, et al. Evidence for intramyocardial disruption of lipid metabolism and increased myocardial ketone utilization in advanced human heart failure. Circulation. 2016;133(8):706-716. doi:10.1161/CIRCULATIONAHA.115.0175 45

9. Lopaschuk GD, Ussher JR. Evolving concepts of myocardial energy metabolism: more than just fats and carbohydrates. Circ Res. 2016;119(11):1173-1176. doi:10.1161/CIRCRESAHA.116.310078

10. Byrne NJ, Soni S, Takahara S, et al. Chronically elevating circulating ketones can reduce cardiac inflammation and blunt the development of heart failure. Circ Heart Fail. 2020;13(6):e006573. doi:10.1161/ CIRCHEARTFAILURE. 119.006573

11. Butler J, Hamo CE, Filippatos G, et al. The potential role and rationale for treatment of heart failure with sodium-glucose co-transporter 2 inhibitors. Eur J Heart Fail. 2017;19(11): 1390-1400. doi:10.1002/ejhf.933

12. Nielsen R, Moller N, Gormsen LC, et al. Cardiovascular effects of treatment with the ketone body 3-hydroxybutyrate in chronic heart failure patients. Circulation. 2019;139(18):2129-2141. doi:10.1161/ CIRCULATIONAHA.118.036459

13. Deng Y, Xie M, Li Q, et al. Targeting mitochondria-inflammation circuit by beta-hydroxybutyrate mitigates HFpEF. Circ Res. 2021;128 (2):232-245. doi:10.1161/CIRCRESAHA.120.317933

14. Schiattarella GG, Altamirano F, Tong D, et al. Nitrosative stress drives heart failure with preserved ejection fraction. Nature. 2019;568(7752):351-356. doi:10.1038/s41586-019-1100-z

15. Liu H, Wang L, Pan Y, et al. Celastrol alleviates aortic valve calcification via inhibition of NADPH oxidase 2 in valvular interstitial cells. JACC Basic Transl Sci. 2020;5(1):35-49. doi:10.1016/j. jacbts.2019.10.004

16. Hirotani S, Zhai P, Tomita H, et al. Inhibition of glycogen synthase kinase 3beta during heart failure is protective. Circ Res. 2007;101 (11):1164-1174. doi:10.1161/CIRCRESAHA.107.160614

17. Horton JL, Davidson MT, Kurishima C, et al. The failing heart utilizes 3-hydroxybutyrate as a metabolic stress defense. JCI Insight. 2019;4(4):e124079. doi:10.1172/jci.insight.124079

18. Aubert G, Martin OJ, Horton JL, et al. The failing heart relies on ketone bodies as a fuel. Circulation. 2016;133(8):698-705. doi:10.1161/CIRCULATIONAHA.115.017355

19. Yurista SR, Matsuura TR, Sillje HHW, et al. Ketone ester treatment improves cardiac function and reduces pathologic remodeling in preclinical models of heart failure. Circ Heart Fail. 2021;14(1): e007684. doi:10.1161/CIRCHEARTFAILURE.120.007684

20. Ho KL, Zhang L, Wagg C, et al. Increased ketone body oxidation provides additional energy for the failing heart without improving cardiac efficiency. Cardiovasc Res. 2019;115(11):1606-1616. doi:10.1093/cvr/cvz045

21. Han YM, Ramprasath T, Zou MH. beta-hydroxybutyrate and its metabolic effects on age-associated pathology. Exp Mol Med. 2020;52(4):548-555. doi:10.1038/s12276-020-0415-z

22. Nagao M, Toh R, Irino Y, et al. beta-Hydroxybutyrate elevation as a compensatory response against oxidative stress in cardiomyocytes. Biochem Biophys Res Commun. 2016;475(4):322-328. doi:10.1016/j. bbrc.2016.05.097

23. Bansal SS, Ismahil MA, Goel M, et al. Activated T lymphocytes are essential drivers of pathological remodeling in ischemic heart failure. Circ Heart Fail. 2017;10(3):e003688. doi:10.1161/ CIRCHEARTFAILURE.116.003688

24. Li N, Bian H, Zhang J, et al. The Th17/Treg imbalance exists in patients with heart failure with normal ejection fraction and heart failure with reduced ejection fraction. Clin Chim Acta. 2010;411(2324):1963-1968. doi:10.1016/j.cca.2010.08.013 
25. Lu M, Qin X, Yao J, et al. Th17/Treg imbalance modulates rat myocardial fibrosis and heart failure by regulating LOX expression. Acta Physiol (Oxf). 2020;230(3):e13537. doi:10.1111/apha.13537

26. Emmerson A, Trevelin SC, Mongue-Din H, et al. Nox2 in regulatory $\mathrm{T}$ cells promotes angiotensin II-induced cardiovascular remodeling. J Clin Invest. 2018;128(7):3088-3101. doi:10.1172/JCI97490

27. Hardt SE, Sadoshima J. Glycogen synthase kinase-3beta: a novel regulator of cardiac hypertrophy and development. Circ Res. 2002;90(10):1055-1063. doi:10.1161/01.res.0000018952.70505.f1
28. Haq S, Choukroun G, Lim H, et al. Differential activation of signal transduction pathways in human hearts with hypertrophy versus advanced heart failure. Circulation. 2001;103(5):670-677. doi:10.11 61/01.cir.103.5.670

29. Matsuda T, Zhai P, Maejima Y, et al. Distinct roles of GSK-3alpha and GSK-3beta phosphorylation in the heart under pressure overload. Proc Natl Acad Sci U S A. 2008;105(52):20900-20905. doi:10.1073/ pnas.0808315106

\section{Publish your work in this journal}

The Journal of Inflammation Research is an international, peerreviewed open-access journal that welcomes laboratory and clinical findings on the molecular basis, cell biology and pharmacology of inflammation including original research, reviews, symposium reports, hypothesis formation and commentaries on: acute/chronic inflammation; mediators of inflammation; cellular processes; molecular mechanisms; pharmacology and novel anti-inflammatory drugs; clinical conditions involving inflammation. The manuscript management system is completely online and includes a very quick and fair peerreview system. Visit http://www.dovepress.com/testimonials.php to read real quotes from published authors. 